ARTICULOS

\title{
¿El sombreamiento es capaz de potenciar el crecimiento de plantas de Myrocarpus frondosus en el campo?
}

\author{
Is shading able to potentiate the growth \\ of Myrocarpus frondosus seedlings in the field?
}

\section{Suelen Carpenedo Aimi a*, Maristela Machado Araujo a , Luciane Almeri Tabaldi ${ }^{\text {b}}$, Enrique Benítez León c, Thairini Claudino Zavistanovicz a, Felipe Manzoni Barbosa a, Álvaro Luis Pasquetti Berghetti a, Marllos Santos de Lima a}

\footnotetext{
*Autor de correspondencia: ${ }^{a}$ Universidade Federal de Santa Maria, Departamento de Ciências Florestais, Av. Roraima 1000, CEP 97105-900, Santa Maria, Brasil, suaimi@gmail.com, araujo.maristela@gmail.com, thairini.z@gmail.com, felipemanzonibarbosa@hotmail.com, alvaro.berghetti@gmail.com, marllos_lima@hotmail.com

b Universidade Federal de Santa Maria, Departamento de Biologia, Santa Maria, Brasil, lutabaldi@yahoo.com.br

${ }^{c}$ Universidad Nacional de Asunción, Facultad de Ciencias Agrarias, Campus San Lorenzo, Paraguay, ebenitezleon@gmail.com
}

\section{SUMMARY}

Light is an important environmental factor in the establishment of vegetation. The knowledge of the behavior of the species in response to light demand for survival and growth in the field becomes essential. Thus, the present study aimed at evaluating the survival and initial growth of Myrocarpus frondosus seedlings under different levels of shade in the field. The treatments used were: $0 \%$ (full sun); $18 \% ; 50 \%$ and $70 \%$ shading in a randomized block design. The survival of seedlings was evaluated 30 and 540 days after planting (d.a.p.) and the morphological attributes height (H), stem diameter (DC) and H / DC ratio every 90 days. Leaf area, leaf dry matter, dry matter of stem and branches and dry matter of shoot were obtained 540 d.a.p. The analyses of the physiological attributes fluorescence of chlorophyll $a$ and photosynthetic pigments were performed 180, 360 and 540 d.a.p. The use of shading in the planting of Myrocarpus frondosus influences the survival and the morphological and physiological attributes of the species. The morphological and physiological attributes of Myrocarpus frondosus plants show that the species requires shading of 50 to $70 \%$ in its initial growth phase in the field (540 d.a.p.). It is recommended to use the species in sub-forest enrichment plantations and in consortium with other species more demanding in light.

Key words: tree species, morphological and physiological attributes, forest enrichment, light demand.

\section{RESUMEN}

La luminosidad es un factor ambiental importante en el establecimiento de la vegetación, siendo imprescindible el conocimiento del comportamiento de las especies en respuesta a la demanda de luz para supervivencia y crecimiento en el campo. El presente estudio tuvo como objetivo evaluar la supervivencia y el crecimiento inicial de plantas de Myrocarpus frondosus bajo diferentes niveles de sombreamiento en el campo. Los tratamientos utilizados fueron $0 \%$ (pleno sol), $18 \%, 50 \%$ y $70 \%$ de sombreamiento en un diseño de bloques al azar. La supervivencia de las plantas fue evaluada a los 30 y 540 días después de la plantación (ddp) y los atributos morfológicos altura (H), diámetro del cuello (DAC) y relación H/DC cada 90 días. A los 540 ddp se obtuvo el área foliar, materia seca foliar, materia seca del tallo y ramas y la materia seca de la parte aérea. Los análisis de los atributos fisiológicos fluorescencia de la clorofila $\alpha$ y los pigmentos fotosintéticos fueron realizados a los 180, 360 y 540 ddp. La utilización de sombreamiento en la plantación de Myrocarpus frondosus evidencian que la especie necesita de 50 y $70 \%$ de sombra en la fase inicial de crecimiento en el campo (540 ddp). Se recomienda el uso de la especie en plantaciones de enriquecimiento de sub bosque y en consorcio con otras especies más exigentes en luminosidad.

Palabras clave: especie forestal, atributos morfológicos y fisiológicos, enriquecimiento forestal, demanda de luz. 


\section{INTRODUCCIÓN}

Un factor importante para el éxito en programas de restauración forestal es la utilización de plantas de calidad de especies adaptadas a las condiciones edafoclimáticas. Las especies poseen adaptaciones fisiológicas complejas que permiten soportar niveles de luminosidad desfavorables, falta de agua y otros factores ambientales (Yu et al. 2018). En ecosistemas naturales la disponibilidad de luz presenta variaciones en el tiempo y en el espacio, siendo muy importante para el establecimiento y crecimiento (Begon et al. 2007, Valladares et al. 2016). Las especies arbóreas presentan respuestas diferentes en relación con la luminosidad, muchas veces asociada al grupo ecológico.

Dependiendo de la condición de luminosidad a que las plantas son expuestas, pueden ocurrir cambios en la morfología foliar para mejorar la eficiencia de captación de luz (Larcher 2000, Martins et al. 2015) siendo eso suficiente para dar continuidad al crecimiento.

Conforme Souza et al. (2009) la capacidad de utilizar y adaptarse a diferentes luminosidades es fundamental para la distribución de las especies en la regeneración de claros. No obstante, poco se conoce sobre las respuestas morfológicas y fisiológicas de las especies arbóreas en diferentes condiciones de luz, observándose que algunas expresan su mejor potencial de crecimiento en pleno sol o en la sombra.

En ese sentido, varias especies con potencial de uso en programas de forestación y reforestación necesitan de investigaciones sobre su desarrollo en diferentes niveles de sombreamiento en el campo, siendo que la mayoría de los estudios fueron desarrollados solamente en la fase de vivero, es decir, en la fase que las plantas son menos susceptibles a daños causados por factores ambientales. Como es el caso de la especie Myrocarpus frondosus Allemão, que no posee informaciones sobre el desarrollo inicial de plantas en relación a la demanda de luz en el campo.

La especie pertenece a la familia Fabaceae, estando presente en las principales formaciones forestales, en Bolivia, Paraguay y Brasil, excepto en el cerrado (Lorenzi 2002). Se destaca entre las principales especies arboles nativas de mayor producción comercial de madera, por ser muy resistente, por la alta densidad y durabilidad, aparte de ser utilizada en la recomposición de ecosistemas alterados, reposición de bosques de galería, arborización urbana, en la industria de perfumerías y medicamentos (Carvalho 2003, Santi et al. 2017). La especie presenta diferentes clasificaciones en cuanto a su grupo sucesional como secundaria inicial (Vaccaro et al. 1999), secundaria tardía (Durigan y Noguera 1990) y semi-heliofita (Carvalho 2003).

Esa divergencia en la clasificación en cuanto al grupo sucesional puede ocasionar problemas en la supervivencia y crecimiento inicial de la especie, evidenciando la necesidad de investigaciones que evalúen su crecimiento y desarrollo en diferentes niveles de sombreamiento, para viabilizar su uso en plantaciones. El trabajo formuló la siguiente hipótesis: en el campo, dentro del área de distribución na- tural de la especie, el sombreamiento facilita el establecimiento y crecimiento de plantas de Myrocarpus frondosus. De esa manera, este estudio tiene como objetivo evaluar la supervivencia y el crecimiento inicial de plantas en campo, bajo diferentes niveles de sombreamiento.

\section{MÉTODOS}

El experimento fue instalado en octubre de 2015, en un área próxima al Vivero Forestal $\left(29^{\circ} 43^{\prime} 13^{\prime \prime} \mathrm{S}\right.$ e $53^{\circ} 43^{\prime}$ 17" O) de la Universidad Federal de Santa Maria (UFSM), municipio de Santa Maria, RS, Brasil, que se encuentra dentro del área de distribución natural de la especie. De acuerdo a la clasificación de Köppen, el clima de la región es subtropical, del tipo "Cfa", con lluvias bien distribuidas durante todos los meses del año, presentando precipitación media anual de $1.620 \mathrm{~mm}$ y temperatura media del mes más frio $-3{ }^{\circ} \mathrm{C}$ y del mes más caliente superior a $22{ }^{\circ} \mathrm{C}$ (Alvares et al. 2013).

Inicialmente fue realizada la colecta de muestras de suelo en las profundidades de 0 a $20 \mathrm{~cm}$ y de 20 a $40 \mathrm{~cm}$, para la caracterización del área, siendo enviadas para el Laboratorio de Análisis de Suelos de la UFSM. De forma general, el análisis de suelo indicó $\mathrm{pH}$ muy bajo a medio $(5,3)$, correspondiendo a un suelo ácido, contenido de materia orgánica (MO) bajo $(1,1 \%)$, nivel de fósforo considerado muy bajo a medio $\left(4,5 \mathrm{mg} \mathrm{dm}^{-3}\right)$, potasio alto $(69,3$ $\left.\mathrm{mg} \mathrm{dm}{ }^{-3}\right)$, magnesio alto $\left(1,8 \mathrm{cmolc}^{-3}\right)$, calcio de medio

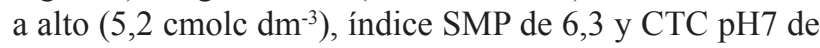
$10,5 \mathrm{cmolc} \mathrm{dm}^{-3}$ (CQFS/SBCS-RS/SC 2004). A pesar del valor bajo de $\mathrm{pH}$ el encalado no fue realizado, teniendo en cuenta que los tenores estaban dentro de los previstos para especies forestales.

El experimento fue conducido en un diseño de bloques al azar, con cuatro repeticiones en cada tratamiento, las evaluaciones se hicieron en diferentes momentos. Cada unidad de muestreo fue representada por seis plantas en espaciamiento $1,0 \mathrm{~m} \times 1,0 \mathrm{~m}$, totalizando 96 plantas. Los niveles del factor de sombreamiento fueron $(0 \%$ - pleno sol, 18,50 y $70 \%$ ) y los niveles del factor tiempo fueron diferentes evaluaciones para atributos morfológicos a los $0,90,180,270,360,450$ y 540 días después de la plantación (ddp), para la supervivencia a los 30 y 540 ddp y atributos fisiológicos a los 180, 360 y 540 ddp. En los tratamientos con sombreamiento fueron utilizadas mallas de nylon, dispuestas sobre arcos de metal recubiertos con caños plásticos con estructura sustentada por estacas de madera. La dimensión de cada armazón fue de 2,5 m x 2,5 $\mathrm{m}$ en la base y 2,20 $\mathrm{m}$ de altura en el área cubierta para no comprometer los tratamientos.

Las plantas de Myrocarpus frondosus utilizadas en la plantación fueron producidas en recipientes de $180 \mathrm{~cm}^{3}$ con sustrato Carolina Soil ${ }^{\circledR}$ a base de turba de Sphagnum y fertilizante de liberación controlada, Osmocote ${ }^{\circledR}, 18-05-$ $09\left(\mathrm{~N}-\mathrm{P}_{2} \mathrm{O}_{5}-\mathrm{K}_{2} \mathrm{O}\right)$ en la dosis de $6 \mathrm{~g} \mathrm{~L}^{-1}$, en el Vivero Forestal de la UFSM. Las mismas fueron seleccionadas de un 
lote que presentó altura promedio de $20,9 \mathrm{~cm}$ y diámetro del cuello de 4,4 mm, 300 días después de la emergencia.

Previamente fue realizada la eliminación semimecanizada (desmalezadora costal) de la vegetación herbácea. La abertura de los hoyos para la plantación fue realizada con la ayuda de un perforador de suelo, acoplado a un tractor, en las dimensiones de $0,3 \mathrm{~m}$ de diámetro $\mathrm{x} 0,3 \mathrm{~m}$ de profundidad $\left(0,02 \mathrm{~m}^{3}\right)$. La fertilización de base fue utilizada con $8 \mathrm{~L}$ de estiércol bovino seco por planta, incorporado al suelo en el hoyo.

Posterior a la plantación, las plantas fueron regadas, para la acomodación del suelo en su entorno. La irrigación de las plantas durante los tres primeros meses fue realizada cada tres días con regaderas, con aproximadamente $2 \mathrm{~L}$ de agua por planta, en la ausencia de precipitación, posterior a ese periodo no hubo más necesidad de irrigaciones, debido a que la precipitación fue suficientemente distribuida para mantener el suelo húmedo.

El control de hormigas cortadoras se realizó cuando fue necesario aplicándose hormiguicida granulado. El control de malezas, alrededor de las plantas, fue realizado con carpida manual (coronado) y entre los bloques con limpieza semimecanizada (desmalezadora costal). La fertilización de cobertura fue realizada a los $180 \mathrm{ddp}$ con $8 \mathrm{~L}$ de estiércol bovino seco por planta, siendo la misma fertilización de base. No obstante, a los 360 ddp, no fue posible obtener el estiércol en el local, así que fue realizada fertilización química con fertilizante de liberación controlada Polyblen ${ }^{\circledR}$ (N-P-K 18-08-18 + azufre y boro), con aproximadamente $130 \mathrm{~g}$ por planta.

La supervivencia de las plantas fue evaluada a los $30 \mathrm{y}$ 540 ddp y a los $0,90,180,270,360,450$ y 540 ddp fueron realizadas las mediciones de los atributos morfológicos altura $(\mathrm{H})$ con regla milimetrada $(\mathrm{cm})$ y diámetro del cuello (DC) con un pie de metro digital de precisión (mm). A partir de esas variables fue posible obtener la relación H/DC.

Además, a los 540 ddp fueron obtenidos los atributos del área foliar (AF), materia seca foliar (MSF), materia seca del tallo y ramas (MSTR) y materia seca de la parte aérea (MSPA). Las colectas fueron realizadas considerando la $\mathrm{H} \mathrm{y}$ el DC promedios de cada repetición, muestreando una planta de cada repetición. Los tallos de las plantas fueron cortados en la altura del cuello, con la ayuda de un serrucho y las hojas fueron separadas del tallo principal y ramas, y utilizadas para la determinación del área foliar. Para AF fueron colectadas aleatoriamente 30 hojas de las plantas, las mismas fueron distribuidas sobre papel A4 con escala milimétrica, prensadas en vidrio y fotografiadas con cámara digital con zoom de 1,4x. Las imágenes fueron procesadas con la ayuda de software Image J. Posteriormente el material fue acondicionado en bolsas de papel tipo Kraft y llevado a una estufa con circulación de aire a $65^{\circ} \mathrm{C}$ hasta alcanzar peso constante $\mathrm{y}$, posteriormente pesadas en balanza analítica, para la determinación de la MSF, MSTR y MSPA. El AF fue obtenida de acuerdo a adaptaciones de la metodología de Coelho-Filho et al. (2012), utilizando la siguiente fórmula:

$$
A F=\frac{(\mathrm{MSTH} * \mathrm{AF} 30 \mathrm{H})}{\mathrm{MS} 30 \mathrm{H}}
$$

En que: $\mathrm{AF}=$ área foliar; $\mathrm{MSTH}=$ materia seca de todas las hojas; $\mathrm{AF} 30 \mathrm{~F}=$ área foliar de 30 hojas; y $\mathrm{MS} 30 \mathrm{H}=$ materia seca de 30 hojas.

Los análisis fisiológicos de fluorescencia de clorofila $\alpha$ y cuantificación de los pigmentos fotosintéticos fueron realizados a los 180,360 y $540 \mathrm{ddp}$ en el campo. La cuantificación de los pigmentos fotosintéticos clorofila $\alpha$, clorofila $b$, relación $\alpha / b$ y carotenoides fue realizada en el Laboratorio de Fisiología Vegetal, del Departamento de Biología de la UFSM. Se colectó la cuarta hoja expandida de tres plantas por tratamiento que fueron congeladas en nitrógeno líquido y, posteriormente, almacenadas en ultra freezer a $-80^{\circ} \mathrm{C}$, hasta la cuantificación. La cuantificación de los pigmentos fotosintéticos fue realizada de acuerdo a la metodología de Hiscox e Israelstam (1979), donde las muestras frescas de hojas $(0,05 \mathrm{~g})$ fueron incubadas a 65 ${ }^{\circ} \mathrm{C}$ con dimetilsulfóxido (DMSO) por dos horas, posteriormente fue realizada la lectura de las absorbancias en espectrofotómetro (Celm E-205D), en las longitudes de onda 663,645 y $470 \mathrm{~nm}$, para Chl $\alpha$, Chl $b$ y carotenoides, respectivamente. Posteriormente las medias fueron estimadas utilizando la fórmula de Lichtenthaler (1987).

La fluorescencia de la clorofila $\alpha$ fue evaluada en cinco plantas por tratamiento, utilizando fluorómetro portátil de luz modulada Junior-Pam (Walz, Germany). La medición fue realizada en días soleados (entre $7 \mathrm{~h} 30 \mathrm{~min}$ y $10 \mathrm{~h} 30 \mathrm{~min}$ ), en el tercio medio de la planta en las hojas expandidas representativas de cada tratamiento. Las hojas fueron adaptadas al oscuro por 30 minutos con papel aluminio, para la medición de la fluorescencia inicial (Fo) y, posteriormente sometidas al pulso de luz saturante $\left(10.000 \mu \mathrm{mol} \mathrm{m}^{-2} \mathrm{~s}^{-1}\right)$ por $0,6 \mathrm{~s}$, determinándose de esta manera la fluorescencia máxima $\left(\mathrm{F}_{\mathrm{m}}\right)$. El rendimiento cuántico máximo PSII $\left(\mathrm{F}_{\mathrm{v}} / \mathrm{F}_{\mathrm{m}}\right)$ fue obtenido por la razón de la fluorescencia variable $\left(\mathrm{F}_{\mathrm{v}}=\mathrm{F}_{\mathrm{m}}-\mathrm{F}_{\mathrm{o}}\right)$ y la fluorescencia máxima. Además de eso, fue obtenida la tasa de transporte de electrones (ETR).

Los datos fueron evaluados en cuanto a los presupuestos de normalidad por el test de Shapiro-Wilk y homogeneidad por el test de Bartlett. Cuando fue observada diferencia significativa entre los tratamientos por el test de $\mathrm{F}$, las medias fueron comparadas por la prueba de Tukey y/o análisis de regresión polinomial, al $5 \%$ de probabilidad de error. Los análisis estadísticos de los datos fueron realizados utilizando el paquete estadístico Sisvar v. 5.3 (Ferreira 2014).

\section{RESULTADOS}

Los datos meteorológicos de precipitación, temperatura media, máxima y mínima registradas en el municipio de Santa María fueron obtenidos en la Estación Meteorológica de Santa María, localizada en el Campus de la UFSM (figura 1). 
Los datos fueron normales y homogéneos. La tasa de supervivencia de las plantas de Myrocarpus frondosus en campo, a los 30 días después de la plantación (ddp) fue de $100 \%$ en los tratamientos 18,50 y $70 \%$ de sombreamiento, difiriendo del tratamiento a pleno sol $(97 \%$ de supervivencia). A los 540 ddp la supervivencia fue menor en los tratamientos a pleno sol $(75 \%)$, difiriendo del sombreamiento con 18 y $50 \%$ (92\%) y del sombreamiento con $70 \%$, que presentó mayor supervivencia (96\%) (cuadro 1).
Se verificó interacción $(P<0,05)$ para los atributos altura $(\mathrm{H})$, diámetro de cuello $(\mathrm{DC})$ y relación H/DC. Para $\mathrm{H}$, el crecimiento fue cuadrático creciente en todos los niveles de sombreamiento (figura $2 \mathrm{~A}$ ), con las mayores medias observadas bajo $70 \%$ y las menores a pleno sol. A los 540 ddp la media de altura fue de $209,7 \mathrm{~cm}$ con $70 \%$ de sombreamiento, mientras que en pleno sol la media fue de 100,1 cm. Para DC también fue observado crecimiento cuadrático creciente, en que las mayores medias fueron

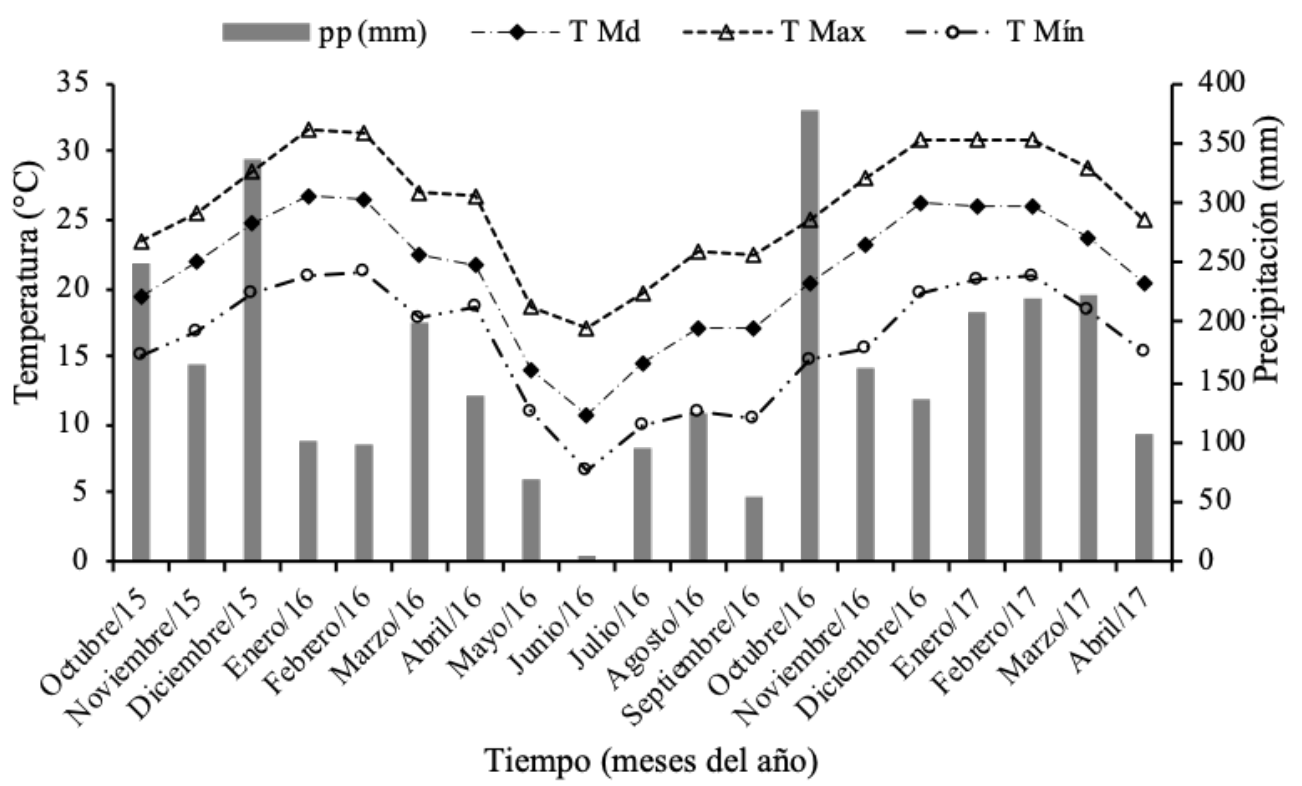

Figura 1. Precipitación (pp), temperatura media (T Md), temperatura máxima ( $\mathrm{T}$ Max) y temperatura mínima ( $\mathrm{T}$ Min) registradas en el municipio de Santa Maria, RS, durante la conducción del experimento de plantas de Myrocarpus frondosus en campo. Fuente: BDMET/INMET (2018).

Precipitation (pp), average temperature (T Md), maximum temperature (T Max) and minimum temperature (T Min) registered in the municipality of Santa Maria, RS, during the conduct of the experiment of seedlings from Myrocarpus frondosus in the field.

Cuadro 1. Tasa de supervivencia, área foliar (AF), materia seca foliar (MSF), materia seca de tallo y ramas (MSTR) y materia seca de la parte aérea (MSPA) de plantas de Myrocarpus frondosus, a los 540 días después de la plantación en campo, Santa María, RS.

Survival rate, leaf area (AF), leaf dry matter (MSF), dry matter of stems and branches (MSTR) and aboveground dry matter (MSPA) plant Myrocarpus frondosus, 540 days after planting in the field, Santa Maria, RS.

\begin{tabular}{|c|c|c|c|c|c|c|}
\hline \multirow{2}{*}{$\begin{array}{c}\text { Nivel de } \\
\text { Sombreamiento (\%) }\end{array}$} & \multicolumn{2}{|c|}{$\begin{array}{c}\text { Supervivencia días después de la } \\
\text { plantación }(\%)\end{array}$} & \multirow{2}{*}{$\begin{array}{l}\mathrm{AF} \\
\left(\mathrm{m}^{2}\right)\end{array}$} & \multirow{2}{*}{$\begin{array}{l}\mathrm{MSF} \\
(\mathrm{g})\end{array}$} & \multirow{2}{*}{$\begin{array}{l}\text { MSTR } \\
(\mathrm{g})\end{array}$} & \multirow{2}{*}{$\begin{array}{l}\text { MSPA } \\
(\mathrm{g})\end{array}$} \\
\hline & 30 & 540 & & & & \\
\hline 0 & $97 b^{*}$ & $75 \mathrm{c}$ & $1,310 b^{*}$ & $115,0 \mathrm{~b}$ & $244,5 \mathrm{~b}$ & $359,5 \mathrm{~b}$ \\
\hline 18 & $100 \mathrm{a}$ & $92 \mathrm{~b}$ & $3,178 \mathrm{a}$ & $192,4 \mathrm{~b}$ & $340,7 \mathrm{~b}$ & $533,0 \mathrm{~b}$ \\
\hline 50 & $100 \mathrm{a}$ & $92 \mathrm{~b}$ & $2,915 \mathrm{a}$ & $257,2 \mathrm{a}$ & $466,8 \mathrm{a}$ & $724,0 \mathrm{a}$ \\
\hline 70 & $100 \mathrm{a}$ & $96 \mathrm{a}$ & $4,368 \mathrm{a}$ & 354,7 a & $648,5 \mathrm{a}$ & $1.003,2 \mathrm{a}$ \\
\hline Media general & 99,25 & 88,7 & 2,94 & 229,8 & 425,1 & 654,9 \\
\hline CV $(\%)$ & 0,88 & 33,46 & 26,04 & 5,96 & 5,20 & 4,77 \\
\hline
\end{tabular}

*Medias seguidas de la misma letra no difieren entre sí, según la prueba de Tukey al 5 \% de probabilidad de error. CV: Coeficiente de variación. 
observadas con $70 \%$ de sombreamiento y las menores en pleno sol (figura 2B). A los 540 ddp las medias fueron de $24,4 \mathrm{~mm}$ en el sombreamiento con $70 \%$ y $19,6 \mathrm{~mm}$ en pleno sol. Para la relación $\mathrm{H} / \mathrm{DC}$ el crecimiento fue cuadrático decreciente en todos los sombreamientos (figura 2C). Las mayores medias fueron observadas con $70 \%$ de sombreamiento y las menores a pleno sol.

Para los atributos área foliar (AF), materia seca foliar (MSF), materia seca del tallo y ramas (MSTR) y materia seca de la parte aérea (MSPA) hubo diferencia $(P<0,05)$

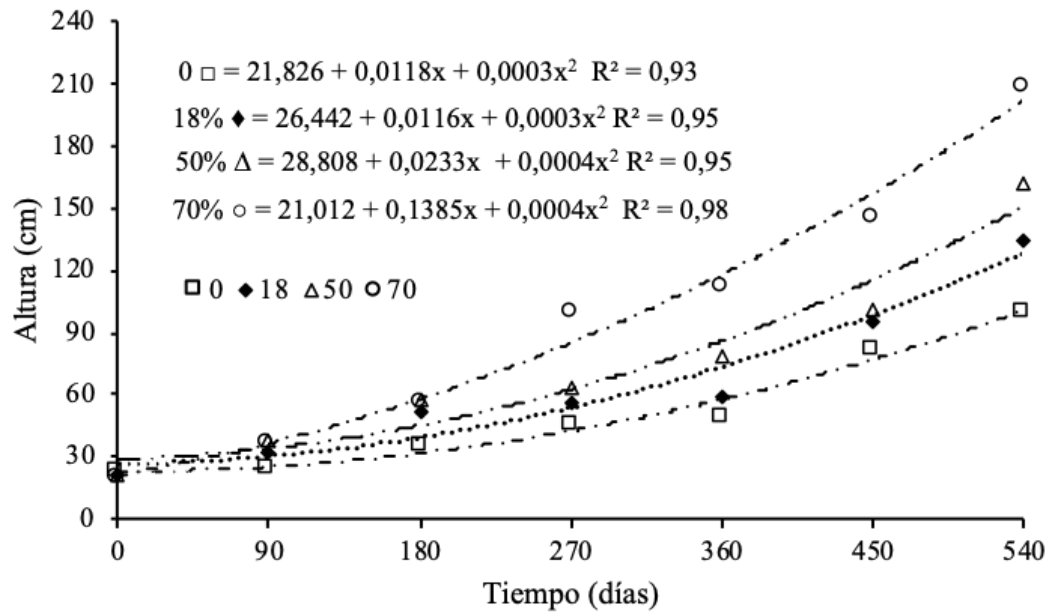

(A)

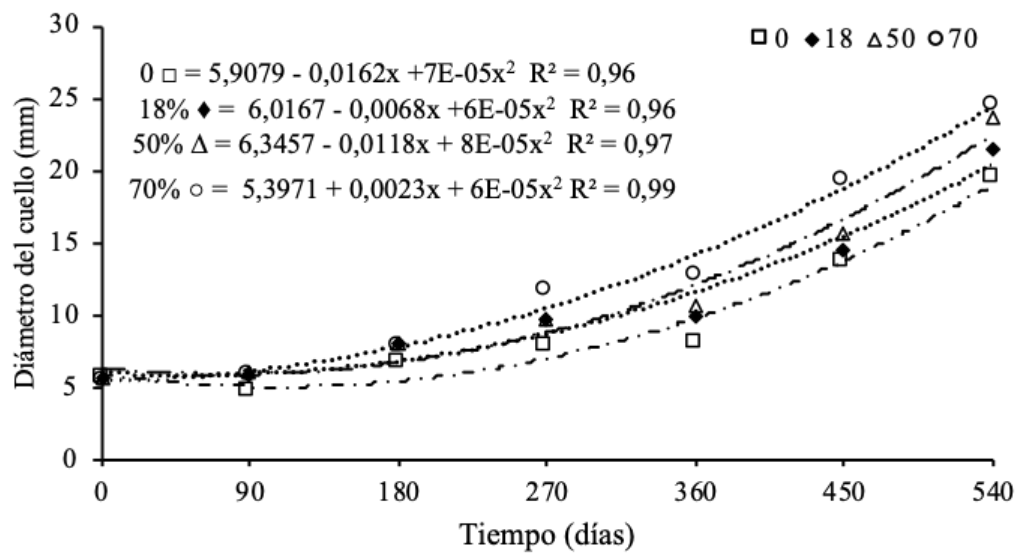

(B)

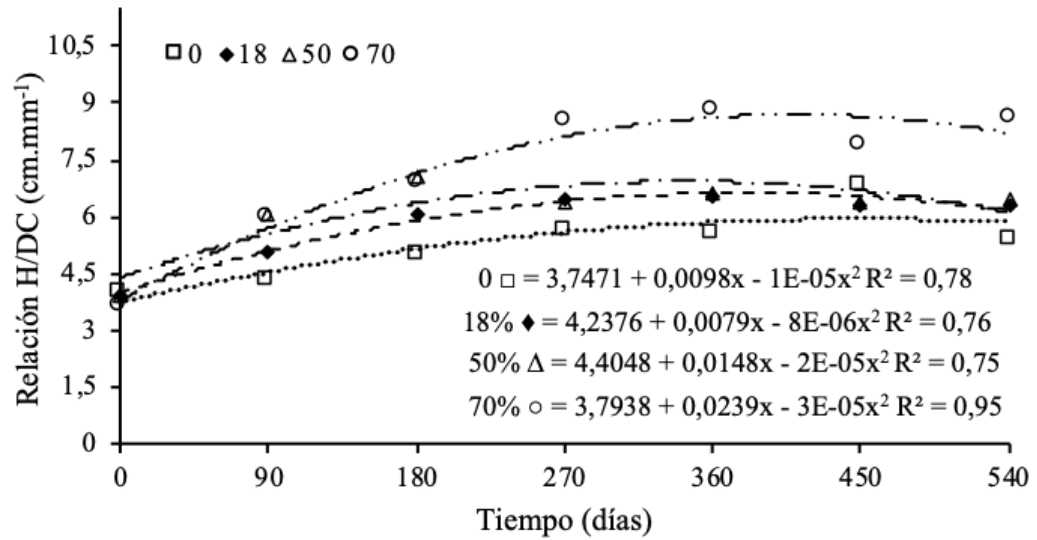

(C)

Figura 2. Crecimiento en altura (A), diámetro de cuello (B) y relación H/DC (C) de plantas de Myrocarpus frondosus, en diferentes intensidades de sombreamiento y tiempos de evaluación, en campo, Santa Maria, RS.

Height growth (A), stem diameter (B) and ratio H/DC (C) seedlings of Myrocarpus frondosus, on different intensity of shading and evaluation times, in the field, Santa Maria, RS. 
entre los sombreamientos. La mayor AF fue observada con $70 \%$ de sombreamiento, no difiriendo de los sombreamientos con 18 y $50 \%$, la menor media fue a pleno sol, difiriendo de los demás sombreamientos (cuadro 1). La MSF, MSTR y la MSPA presentan comportamientos semejantes en los diferentes sombreamientos, las mayores medias fueron encontradas en el sombreamiento con $70 \%$, no difiriendo del tratamiento con $50 \%$ y las menores medias fueron observadas en el tratamiento a pleno sol.

Para los atributos clorofila $\alpha$, clorofila $b$ y razón $\alpha / b$ no hubo interacción, solamente efecto significativo aislado para los factores. La tasa de transporte de electrones no presentó diferencia significativa para el sombreamiento $(P=0,9944)$ apenas para el tiempo a los 360 ddp. La clorofila $\alpha$ fue mayor a 50 y $70 \%$ de sombreamiento, difiriendo los demás tratamientos y la menor media fue a pleno sol (cuadro 2). En el tiempo, las mayores medias fueron observadas a los 180 y 360 ddp. Para la clorofila $b$ la mayor media fue observada con $70 \%$ de sombreamiento y en las evaluaciones a los 180 y 360 ddp. La razón clorofila $\alpha / b$ también presentó la misma tendencia en el tiempo, no obstante, las mayores medias fueron observadas con $50 \mathrm{y}$ $70 \%$ de sombreamiento. Para los carotenoides no hubo diferencia para el sombreamiento $(P=0,0988)$, solo para los tiempos de evaluación, siendo que la mayor media fue observada a los $540 \mathrm{ddp}$.

Hubo interacción $(P<0,05)$ entre los factores sombreamiento $\mathrm{x}$ tiempo para la fluorescencia inicial $\left(\mathrm{F}_{\mathrm{o}}\right)$, fluorescencia máxima $\left(\mathrm{F}_{\mathrm{m}}\right)$ y rendimiento cuántico máximo PSII $\left(\mathrm{F}_{\mathrm{v}} / \mathrm{F}_{\mathrm{m}}\right)$. Para Fo hubo solo diferencia en el sombreamiento con $50 \%$ y $70 \%$ a los 540 ddp (cuadro 3).
Con relación a los tiempos de evaluación las mayores medias fueron observadas a los $540 \mathrm{ddp}$ para pleno sol, $50 \%$ y $70 \%$ de sombreamiento, no difiriendo de $18 \%$ a los 180 ddp. Para $\mathrm{F}_{\mathrm{m}}$ las mayores medias fueron observadas a los 180 y $540 \mathrm{ddp}$ a pleno sol y con $18 \%$ de sombreamiento no difiriendo de los sombreamientos a los $540 \mathrm{ddp}$. Las mayores medias de $\mathrm{F}_{\mathrm{v}} / \mathrm{F}_{\mathrm{m}}$ fueron observadas a los $360 \mathrm{ddp}$ (cuadro 3).

\section{DISCUSIÓN}

En el establecimiento inicial a campo de las plantas de Myrocarpus frondosus es necesario sombreamiento para que ocurra mayor supervivencia (cuadro 1). La tasa de supervivencia inicial observada puede ser considerada alta para especies nativas, demostrando adecuado establecimiento en campo. Resultados semejantes fueron observados en estudios con sombreamiento con Apuleia leiocarpa (Vogel) J.F. Macbr (Aimi et al. 2017). En ese sentido, se destaca las variaciones de las especies arbóreas, en cuanto a condiciones de sombreamiento, y la necesidad de estudios considerando la luminosidad como un factor de selección en el bosque, durante los estadios sucesionales (Freitas et al. 2012).

Para los atributos morfológicos $\mathrm{H}, \mathrm{DC}$, y relación $\mathrm{H} /$ DC fueron observadas las mayores medias para plantas sometidas a $70 \%$ de sombreamiento (figura 2). A los $540 \mathrm{ddp}$ las plantas presentaron media de $\mathrm{H}$ de $210 \mathrm{~cm}$, lo que está de acuerdo con Lorenzi (2002) que describe a la especie con crecimiento medio de $2,5 \mathrm{~m}$ a los dos años. Además de eso, cuanto mayor la H y el DC, mejor será la supervivencia y el establecimiento de las plantas en campo.

Cuadro 2. Tasa de transporte de electrones (ETR), clorofila $\alpha$, clorofila $b$, razón clorofila $\alpha$ /clorofila $b$ y carotenoides de plantas de Myrocarpus frondosus en diferentes sombreamientos y tiempos de evaluación en campo, Santa Maria, RS.

Electron transport rate (ETR), chlorophyll $a$, chlorophyll $b$, chlorophyll $a$ /chlorophyll $b$ and carotenoids Myrocarpus frondosus seedlings in different shades and evaluation times in the field, Santa Maria, RS.

\begin{tabular}{|c|c|c|c|c|c|}
\hline $\begin{array}{c}\text { Nivel de } \\
\text { sombreamiento (\%) }\end{array}$ & $\begin{array}{l}\text { Tasa de transporte } \\
\text { de electrones - ETR } \\
\left(\mu \mathrm{mol} \mathrm{m} \mathrm{m}^{-2} \mathrm{~s}^{-1}\right)\end{array}$ & $\begin{array}{l}\text { Clorofila } a \\
\left(\mathrm{mg} \mathrm{g}^{-1}\right)\end{array}$ & $\begin{array}{l}\text { Clorofila } b \\
\left(\mathrm{mg} \mathrm{g}^{-1}\right)\end{array}$ & $\begin{array}{l}\text { Razón } \\
\text { clorofila } a / b\end{array}$ & $\begin{array}{c}\text { Carotenoides } \\
\left(\mathrm{mg} \mathrm{g}^{-1}\right)\end{array}$ \\
\hline 0 & $139,353^{\mathrm{ns}}$ & $1,357 \mathrm{~b}$ & $0,411 \mathrm{~b}$ & $3,181 \mathrm{~b}$ & $0,436^{\mathrm{ns}}$ \\
\hline 18 & 139,799 & $1,402 \mathrm{~b}$ & $0,417 \mathrm{~b}$ & $3,272 \mathrm{~b}$ & 0,444 \\
\hline 50 & 133,466 & $1,530 \mathrm{a}$ & $0,506 \mathrm{~b}$ & $3,672 \mathrm{a}$ & 0,480 \\
\hline 70 & 135,864 & $1,550 \mathrm{a}$ & $0,615 \mathrm{a}$ & $3,921 \mathrm{a}$ & 0,474 \\
\hline \multicolumn{6}{|l|}{ Tiempo (días) } \\
\hline 180 & $77,242 b^{*}$ & $1,569 \mathrm{a}$ & 0,583 a & $3,892 \mathrm{a}$ & $0,446 \mathrm{~b}$ \\
\hline 360 & $265,598 \mathrm{a}$ & $1,556 \mathrm{a}$ & $0,530 \mathrm{a}$ & $3,765 \mathrm{a}$ & $0,444 \mathrm{~b}$ \\
\hline 540 & $68,521 \mathrm{~b}$ & $1,253 \mathrm{~b}$ & $0,348 \mathrm{~b}$ & $2,878 \mathrm{~b}$ & $0,486 \mathrm{a}$ \\
\hline Media general & 137,120 & 1,459 & 0,487 & 3,511 & 0,459 \\
\hline CV $(\%)$ & 2,77 & 12,17 & 29,15 & 15,57 & 10,83 \\
\hline
\end{tabular}

"Medias seguidas de la misma letra no difieren entre sí, según la prueba de Tukey al $5 \%$ de probabilidad de error. ${ }^{\text {ns }}=$ no significativo y CV: Coeficiente de variación. 
La mayor capacidad de crecimiento de las plantas en ambientes sombreados es un mecanismo importante de adaptación de la especie para sobrevivir en esa condición de menor intensidad luminosa (Siebeneichlen et al. 2008). Cuando eso ocurre, no necesariamente significa que la planta demanda sombreamiento durante la plantación, pero que hasta determinado punto responde favorablemente a tal condición, como fue observada para las plantas de M. frondosus a los $540 \mathrm{ddp}$. A partir de eso, otros atributos morfológicos evaluados pueden ser utilizados para verificar la calidad de las plantas, lo que puede ser comprobado con el AF y MSPA.

Las medias de los atributos morfológicos en los mayores niveles de sombreamiento $(50 \%$ y $70 \%$ ) demostraron que las plantas de M. frondosus no presentan etiolamiento, que es una respuesta morfogénica que ocurre por la menor condición de luminosidad durante el crecimiento vegetal e incluye el alargamiento caulinar envés de aumentar en espesor (Taiz et al. 2017). Además de eso, esa alteración ocurre principalmente en las especies arbóreas con poca tolerancia al sombreamiento en el bosque, que de esa forma alcanzan, con mayor rapidez, superar en altura las plantas que las sombrean (Poorter 1999).

El AF y la MSA también fueron mayores en el sombreamiento con $50 \%$ y $70 \%$ lo que puede ser explicado por el hecho de la necesidad de aumentar la captación de luz ocasionando hojas con área foliar mayor (Larcher y Boeger 2009). Generalmente, el incremento del AF con el sombreamiento es una de las maneras de la planta aumentar a superficie fotosintética, asegurando un aprovechamiento más eficiente de las menores intensidades luminosas y, compensando las bajas tazas de fotosíntesis en las hojas de sombra (Jones y McLeod 1990). En pleno sol fueron encontradas las menores medias para AF y MSA, así la mayor disponibilidad de luz en las hojas se refleja en el espesamiento y menor área foliar, pues la luz no es un factor limitante (Larcher y Boeger 2009). El aumento en la espesura de la hoja en especies en pleno sol ocurre debido a la mayor cantidad de células que forman la camada empalizada, protegiendo la hoja en contra

Cuadro 3. Fluorescencia inicial $\left(\mathrm{F}_{\mathrm{o}}\right)$, fluorescencia máxima $\left(\mathrm{F}_{\mathrm{m}}\right)$ y rendimiento cuántico máximo $\left(\mathrm{F}_{\mathrm{v}} / \mathrm{F}_{\mathrm{m}}\right)$ de plantas de Myrocarpus frondosus en diferentes sombreamientos y tiempos de evaluación en campo, Santa Maria, RS.

Initial fluorescence $\left(\mathrm{F}_{\mathrm{o}}\right)$, maximum fluorescence $\left(\mathrm{F}_{\mathrm{m}}\right)$ and maximum PSII in quantum yield $\left(\mathrm{F}_{\mathrm{v}} / \mathrm{F}_{\mathrm{m}}\right)$ of seedlings from Myrocarpus frondosus in the field in different shades and times, Santa Maria, RS.

\begin{tabular}{|c|c|c|c|c|}
\hline \multirow{2}{*}{ Atributos } & \multirow{2}{*}{$\begin{array}{c}\text { Nivel de } \\
\text { Sombreamento (\%) }\end{array}$} & \multicolumn{3}{|c|}{ Tiempo (días) } \\
\hline & & 180 & 360 & 540 \\
\hline \multirow{4}{*}{$\mathrm{F}_{\mathrm{o}}$} & 0 & $192,0 \mathrm{Ba}^{*}$ & $153,5 \mathrm{Ba}$ & $244,2 \mathrm{Ab}$ \\
\hline & 18 & 226,7 Aa & $142,25 \mathrm{Ba}$ & $309,5 \mathrm{Ab}$ \\
\hline & 50 & $240,7 \mathrm{Ba}$ & $154,5 \mathrm{Ca}$ & $460,0 \mathrm{Aa}$ \\
\hline & 70 & $177,7 \mathrm{Ba}$ & $157,25 \mathrm{Ba}$ & $537,3 \mathrm{Aa}$ \\
\hline Media general & \multicolumn{4}{|c|}{216,31} \\
\hline CV (\%) & \multicolumn{4}{|c|}{6,76} \\
\hline \multirow{4}{*}{$\mathrm{F}_{\mathrm{m}}$} & 0 & $533,5 \mathrm{Aa}$ & $186,5 \mathrm{Ba}$ & $353,2 \mathrm{Ac}$ \\
\hline & 18 & 544,0 Aa & $184,0 \mathrm{Ba}$ & $705,0 \mathrm{Ab}$ \\
\hline & 50 & $591,5 \mathrm{Ba}$ & $238,7 \mathrm{Ca}$ & $919,0 \mathrm{Aa}$ \\
\hline & 70 & $561,5 \mathrm{Ba}$ & $248,0 \mathrm{Ca}$ & $1165,0 \mathrm{Aa}$ \\
\hline Media general & \multicolumn{4}{|c|}{519,1} \\
\hline CV (\%) & \multicolumn{4}{|c|}{5,48} \\
\hline \multirow{4}{*}{$\mathrm{F}_{\mathrm{v}} / \mathrm{F}_{\mathrm{m}}$} & 0 & $0,73 \mathrm{Aa}$ & $0,71 \mathrm{Aa}$ & $0,31 \mathrm{Bb}$ \\
\hline & 18 & $0,59 \mathrm{Ba}$ & $0,78 \mathrm{Aa}$ & $0,53 \mathrm{Ba}$ \\
\hline & 50 & $0,60 \mathrm{Ba}$ & $0,76 \mathrm{Aa}$ & $0,45 \mathrm{Ca}$ \\
\hline & 70 & $0,69 \mathrm{Ba}$ & $0,78 \mathrm{Aa}$ & $0,54 \mathrm{Ba}$ \\
\hline Media general & \multicolumn{4}{|c|}{0,62} \\
\hline CV (\%) & \multicolumn{4}{|c|}{15,26} \\
\hline
\end{tabular}

*Medias seguidas de la misma letra mayúscula en la línea y minúsculas en la columna no difieren entre sí, según la prueba de Tukey al $5 \%$ de probabilidad de error. CV: Coeficiente de variación. 
de posibles daños causados por la elevada luminosidad (Taiz et al. 2017).

Para la clorofila $\alpha$ y clorofila $b$ las mayores medias fueron observadas con $50 \%$ y $70 \%$ de sombreamiento. De forma general, las clorofilas y los carotenoides tienden a aumentar con la reducción de la intensidad luminosa. La clorofila $\alpha$ es el principal pigmento utilizado en la etapa fotoquímica de la fotosíntesis, pues constituye el centro de reacción del fotosistema, en cuanto a la clorofila $b$ actúa en el complejo antena colector de luz, auxiliando en la observación y transferencia de energía para el PSII (Streit et al. 2005). El aumento de las clorofilas en los niveles de mayor sombreamiento puede ser debido a la compensación de la especie a la menor cantidad de radiación disponible. En hojas sombreadas, como es el caso de las especies no pioneras en su estadio inicial de crecimiento, puede ocurrir alargamiento del parénquima lagunoso. También, puede ocurrir aumento en la síntesis de clorofila $b$ y carotenoides, intensificando la capacidad de absorción de luz en el ambiente sombreado (Taiz et al. 2017).

Para los carotenoides no hubo diferencia entre los sombreamientos, apenas para el tiempo (cuadro 2). Normalmente, los carotenoides tienden a aumentar con la reducción de la intensidad luminosa y durante la fotosíntesis pueden desempeñar dos funciones distintas: absorción de luz (pigmentos accesorios) y foto protectores del aparato fotoquímico (Kerbauy 2004). Conforme Streit et al. (2005) por la estructura química ser inestable, las clorofilas son fácilmente degradadas y sintetizadas, en la misma proporción. Así, hojas de sombra presentan mayor cantidad de pigmentos que hojas a pleno sol.

La menor media de la fluorescencia inicial $\left(\mathrm{F}_{\mathrm{o}}\right)$, obtenida con $70 \%$ de sombreamiento a los 180 ddp con $18 \%$ de sombreamiento a los $360 \mathrm{ddp}$ y a pleno sol a los $540 \mathrm{ddp}$ demuestran plantas en mejores condiciones en relación a los otros sombreamientos, pues cuanto menor el valor de $\mathrm{F}_{\mathrm{o}}$, menor es la pérdida de energía. El aumento en los valores de $\mathrm{F}_{\mathrm{o}}$ a los 180 y 540 ddp indica disminución en la capacidad de transferencia de energía del complejo antena para los centros de reacciones (Baker y Rosenqvist 2004). Además, el aumento en la $\mathrm{F}_{\mathrm{o}}$ indica que hubo daño en la proteína D1 del centro de reacción del PSII, ocasionando daños irreversibles al complejo de evolución de oxígeno (Bertamini et al. 2004).

Con relación a la fluorescencia máxima $\left(\mathrm{F}_{\mathrm{m}}\right)$, a los 180 ddp esos valores permanecieron próximos en todos los sombreamientos, pero con el correr del tiempo hubo alteraciones y las menores medias fueron observadas con $18 \%$ de sombreamiento y a pleno sol, principalmente a los 540 ddp. La reducción de $\mathrm{F}_{\mathrm{m}}$ ha sido atribuida a la disipación no fotoquímica, principalmente por medio del ciclo de la xantofila. De ese modo, el exceso de energía absorbida por la hoja es drenado para los carotenoides del ciclo de la xantofila, que la disipa en la forma de calor, protegiendo el fotosistema II contra posibles daños oxidativos causados por la exposición a pleno sol (Demmig-Adams y Adams 2006).
La reducción de $\mathrm{F}_{\mathrm{v}} / \mathrm{F}_{\mathrm{m}}$ puede estar asociada al tiempo de evaluación, pues a los 540 ddp correspondió al mes de abril, es decir, posterior al término del verano, periodo con menor incidencia de luz y temperaturas (Figura 1). Esa reducción indicó menor cantidad de energía absorbida por los pigmentos fotosintéticos en el complejo ante de la planta, que es utilizada para reducir el carbono y producir materia seca. En plantas saludables esa relación debe estar entre 0,75 y 0,85 , valores inferiores a 0,75 indican reducción del potencial fotosintético de la planta en función de alguna situación de estrés (Ritchie et al. 2010).

En plantas de la especie Cordia superba Cham. con 12 meses de edad, en dos ambientes con diferentes luminosidades (pleno sol y $85 \%$ de sombreamiento), Souza et al. (2009) verificaron que las hojas en el ambiente sombreado mostraron mayores valores para $\mathrm{F}_{\mathrm{v}} / \mathrm{F}_{\mathrm{m}}$ que hojas a pleno sol, pudiendo ser considerada una característica deseable en ambiente con baja disponibilidad de luz. Disminución del $F_{v} / F_{m}$ posterior a la exposición a alta irradiación de las plantas aclimatadas a condición de sombreamiento también fueron observados en otras especies arbóreas (Tobita et al. 2010, Aimi et al. 2017). La reducción del valor de ETR a los 540 ddp puede indicar disminución en la actividad del fotosistema II, con el aumento de la fotoinhibición.

Myrocarpus frondosus es una planta decidua (Lorenzi 2002), lo que puede explicar esa variación en las medias de fluorescencia de la clorofila $\alpha$. A los 360 ddp las evaluaciones fueron realizadas en octubre (invierno-primavera). Mientras que las evaluaciones a los 180 y 540 ddp fueron realizadas en abril (verano-otoño). Las plantas deciduas son aquellas que evolutivamente fueron sensibles a la reducción del fotoperiodo y desencadenan mecanismos de abscisión de las hojas en el invierno (Larcher 2000). Con el aumento del periodo de luz en la primavera, las plantas liberan las hojas nuevas producidas en el final del otoño y mantenidas protegidas por brácteas durante todo el invierno, iniciando un nuevo periodo de primavera y verano de intensa actividad fotosintética (Taiz et al. 2017). En este estudio no fue observado pérdida de hojas de las plantas de M. frondosus, no obstante, a los 360 ddp hubo mayor taza de transporte de electrones y relación $\mathrm{F}_{\mathrm{v}} / \mathrm{F}_{\mathrm{m}}$.

En ambientes forestales, el aparato fotosintético debe ser capaz de utilizar la energía luminosa disponible de forma eficiente, una vez que esa disponibilidad se altera de acuerdo al periodo de exposición y la intensidad luminosa (Way y Pearcy 2012). Conforme Taiz et al. (2017) esa utilización eficiente de la luz está relacionada al mantenimiento de la capacidad de activación del aparato fotosintético, y envuelve factores bioquímicos y estomáticos, una vez que la luz actúa en la activación de enzimas relacionadas a la fijación de carbono y en el control de abertura y cierre de estomas.

Myrocarpus frondosus es clasificada como secundaria inicial (Vaccaro et al. 1999) a secundaria tardía (Durigan y Nogueira 1990) y semi-heliofita que tolera sombreamiento de media intensidad (Carvalho 2003), esa plasticidad po- 
siblemente esté asociada a su amplia distribución geográfica. Mientras tanto, en regiones con las cuatro estaciones bien definidas, la especie presentó mejor crecimiento con 50 y $70 \%$ de sombreamiento. Así, se recomienda el uso de la especie en plantaciones de enriquecimiento del subbosque y en asociación con otras especies.

\section{CONCLUSIONES}

La hipótesis planteada: en el campo, el sombreamiento facilita el establecimiento y crecimiento de plantas de Myrocarpus frondosus, es aceptada.

La utilización de sombreamiento en la plantación de Myrocarpus frondosus favoreció la supervivencia de las plantas y a los atributos morfológicos y fisiológicos.

Los atributos morfológicos y fisiológicos de las plantas evidenciaron que la especie necesita de sombreamiento de 50 a $70 \%$ en la fase inicial de su crecimiento en campo (540 ddp).

\section{AGRADECIMIENTOS}

A la Coordinación de Perfeccionamiento de Personal de Nivel Superior (CAPES) por la concesión de la beca de doctorado de la primera autora, al Consejo Nacional de Desarrollo Científico y Tecnológico $(\mathrm{CNPq})$ por la beca de productividad científica de la segunda autora. Al señor Élio Campanhol por la ayuda brindada en la ejecución del experimento.

\section{REFERENCIAS}

Aimi SC, MA Araujo, ST Tonetto, LA Tabaldi, CS Witt, JF Gomes, GG Oliveira. 2017. Shading as a conditioning factor to forest species planting: a study with Apuleia leiocarpa. Bosque 38(2): 371-379. DOI: 10.4067/S071792002017000200014

Alvares CA, JL Stape, PC Sentelhas, JLM Gonçalves, G Sparovek. 2013. Köppen's climate 353 classification map for Brazil. Meteorologische Zeitschrift 2: 1-18. DOI: 10.1127/0941-2948/2013/0507

Baker N, E Rosenqvist. 2004. Applications of chlorophyll fluorescence can improve crop production strategies: an examination of future possibilities. Journal of Experimental Botany 55(403): 1607-1621. DOI: 10.1093/jxb/erh196

Begon M, CR Towsend, JL Harper. 2007. Ecologia: de indivíduos a populações. Porto Alegre. Artmed. 752 p.

Bertamini M, K Muthuchelian, N Nedunchezhian. 2004. Photoinhibition of photosynthesis in sun and shade grown leaves of grapevine (Vitis vinifera L.). Photosynthetica 42: 7-14. DOI: 10.1023/B:PHOT.0000040563.64215.63

Carvalho PER. 2003. Espécies Arbóreas Brasileiras. Brasília. EMBRAPA Informação Tecnológica. 1039 p.

Coelho-Filho MA, NA Villa-Nova, LR Angelocci, FR Marin, CA Righi. 2012. Método para estimativa do IAF de árvores isoladas ou de plantações com dossel fechado. Revista Brasileira de Engenharia Agrícola e Ambiental 16(5): 529-538. DOI: $\underline{10.1590 / \text { S1415-43662012000500009. }}$.

CQFS/SBCS (Comissão de Química e Fertilidade do Solo, So- ciedade Brasileira de Ciência do Solo, BR). 2004. Manual de adubação e de calagem para os estados do RS e SC. 10 ed. Porto Alegre. Sociedade Brasileira de Ciência do Solo Núcleo Regional Sul. 394 p.

Demmig-Adams B, WW Adams. 2006. Photoprotection in an ecological context: the remarkable complexity of thermal energy dissipation. New Phytologist 172: 11-21. DOI: 10.1111/j.1469-8137.2006.01835.x

Durigan G, JCB Nogueira. 1990. Recomposição de matas ciliares. São Paulo. Instituto Florestal. 14 p.

Ferreira DF. 2014. Sisvar: a guide for its bootstrap procedures in multiple comparisons. Ciência e Agrotecnologia 38(2): 109-112. DOI: $10.1590 /$ S1413-70542011000600001

Freitas GA, A Vaz-de-Melo, MAB Pereira, CAO Andrade, GN Lucena, RR da Silva. 2012. Influência do sombreamento na qualidade de mudas de Sclerolobium paniculatum Vogel para recuperação de área degradada. Journal of Biotechnology and Biodiversity 3(3): 5-12. DOI: https://doi. org/10.20873/jbb.uft.cemaf.v3n3.freitas

Hiscox JD, GF Israelstam. 1979. A method for the extraction of chlorophyll from leaf tissue without maceration. Canadian Journal of Botany 57(12): 1132-1334. DOI: 10.1139/b79-163

Jones RH, KW Mcleod. 1990. Responses to a range o light environments in Chinese Tallowtree and Carolina Ash seedlings. Forest Science 36: 851-862.

Kerbauy GB. 2004. Fisiologia Vegetal. Rio de Janeiro. Guanabara Koogan. 472 p.

Larcher W. 2000. Ecofisiologia Vegetal. São Carlos, Brasil. Editora Rima. $531 \mathrm{p}$.

Larcher L, MRT Boeger. 2009. Arquitetura foliar de Odontonema strictum (Nees) O. Kuntze (Acanthaceae) em duas condições de luminosidade. Hoehnea 36(2): 321-327. DOI: http://dx.doi.org/10.1590/S2236-89062009000200009

Lichtenthaler HK. 1987. Chlorophylls and carotenoids: pigments of photosynthetic biomembranes. Methods in Enzymology 148: 350-382. DOI: 10.1016/0076-6879(87)48036-1

Lorenzi H. 2002. Árvores Brasileiras. Manual de identificação e cultivo de plantas arbóreas nativas do Brasil. Nova Odessa, São Paulo. Plantarum. 378 p.

Martins ACF, IA Schiavini, M de Glein, S de F Lopes. 2015. Capacidade adaptativa de espécies do cerrado utilizadas em áreas de recuperação ambiental. Revista Árvore 39(3): 543550. DOI: $10.1590 / 0100-67622015000300015$

Poorter L. 1999. Growth responses of 15 rain-forest tree species to a light gradient: the relative importance of morphological and physiological traits. Functional Ecology 13: 396-410. DOI: $10.1046 / \mathrm{j} .1365-2435.1999 .00332 . x$

Ritchie GA, TD Landis, RK Dumroese, DL Haase. 2010. Assessing plant quality. Seedling Processing, Storage, and Outplanting. Washington DC. Department of Agriculture Forest Service. 200 p. (Agriculture Handbook 674)

Santi II de, DA Gatto, MRG Machado, PSB dos Santos, RA Freitag. 2017. Chemical Composition, Antioxidant and Antimicrobial Activity of the Oil and Plant Extract Myrocarpus frondosus Allemão. American Journal of Plant Sciences 8: 1560-1571. DOI: 10.4236 /ajps.2017.87108

Souza GM, BD Balmant, HF Vítolo, KBP Gomes, TM Florentino, TA Catuchi. 2009. Estratégias de utilização de luz e estabilidade do desenvolvimento de plântulas de Cordia superba Cham. (Boraginaceae) crescidas em diferentes ambientes luminosos. Acta Botânica Brasílica 23: 474-485. 
DOI: 10.1590/S0102-33062009000200019

Streit NM, LP Canterle, MW do Canto, LHH Hecktheuer. 2005. As clorofilas. Ciência Rural 35(3): 748-755. DOI: $10.1590 /$ $\underline{\mathrm{S} 0103-84782005000300043}$

Taiz L, E Zeiger, I Moller, A Murphy. 2017. Fisiologia e desenvolvimento vegetal. Porto Alegre. Artmed. 888 p.

Tobita H, H Utsugi, M Kitao, M Kayama, A Uemura, S Kitaoka, Y Maruyama. 2010. Variation in photoinhibition among Sasa senanensis, Quercus mongolica, and Acer mono in understory of a deciduous broad-leaved Forest exposed to canopy gaps caused by typhoons. Trees 24: 307-319. DOI: 10.1007/s00468-009-0400-6

Vaccaro S, SJ Longhi, DA Brena. 1999. Aspectos da composição florística e categorias sucessionais do estrato arbóreo de três sub seres de uma floresta estacional decidual, no $\mathrm{Mu}-$ nicípio de Santa Tereza - RS. Ciência Florestal 9(1): 1-18. DOI: $10.5902 / 19805098360$

Valladares F, L Laanisto, Ü Niinemets, MA Zavala. 2016. Shedding light on shade: ecological perspectives of understorey plant life. Plant Ecology \& Diversity 9: 237-251. DOI: 10.1080/17550874.2016.1210262

Way DA, RW Pearcy. 2012. Sunflecks in trees and forests: from photosynthetic physiology to global change biology. Tree Physiology 32(9): 1066-1081. DOI: 10.1093/treephys/tps064

Yu Q, Y Shen, Q Wang. 2018. Light deficiency and water logging affect chlorophyll metabolism and photosynthesis in Magnolia sinostellata. Trees 33: 11-22. DOI: $\underline{10.1007 / \mathrm{s} 00468-}$ $\underline{018-1753-5}$

Recibido: 23/09/19

Aceptado: 06/04/20 